\title{
Increased Bilateral Frontal Connectivity during Working Memory in Young Adults under the Influence of a Dopamine D1 Receptor Antagonist
}

\author{
Anna Rieckmann, ${ }^{1}$ Sari Karlsson, ${ }^{1}$ Håkan Fischer, ${ }^{2}$ and Lars Bäckman ${ }^{1}$ \\ ${ }^{1}$ Aging Research Center, Karolinska Institute, SE-113 30 Stockholm, Sweden, and 2Department of Psychology, Stockholm University, SE-106 91 Stockholm, \\ Sweden
}

Increased frontal bilaterality in old compared with young adults during cognitive performance is a common finding in human functional neuroimaging studies. Age-related reductions in laterality are a widely debated topic and their origins and consequences may be manifold. The current study demonstrates that a dopamine (DA) D1 antagonist induces increased frontal bilateral connectivity in healthy young adults revealed by functional magnetic resonance imaging during a spatial working memory task. Moreover, increases in functional connectivity between right and left prefrontal cortex during the pharmacological challenge were associated with maintaining performance on drug. To our knowledge, this is the first study to pharmacologically induce increased frontal bilateral functional connectivity during a cognitive task in young adults and to show that increased bilaterality is associated with less severe cognitive impairment under the influence of a DA receptor antagonist.

\section{Introduction}

Age-related decreases in frontoparietal activation and functional connectivity during working memory (WM) are common in neuroimaging studies and likely reflect reduced integrity of neural networks (Goh, 2011; Nagel et al., 2011; Rieckmann et al., 2011). At the same time, functional magnetic resonance imaging (fMRI) during WM often reveals an age-related increase in frontal bilaterality, both in terms of activation and connectivity (Spreng et al., 2010; Goh, 2011). Increased frontal bilaterality in old adults has been related to better cognitive performance (Grady et al., 2005; Gutchess et al., 2005). However, other studies have not reported such a link and the functional relevance and neural underpinnings of increased frontal bilaterality in aging remain widely debated (Rajah and D'Esposito, 2005; Spreng et al., 2010; Morcom and Friston, 2012). A recent study showed that increased frontal activation in old adults was found in a crosssectional comparison only. When old adults were followed over 6 years they exclusively showed decreases in task-relevant areas (Nyberg et al., 2010). This suggests that age-comparative studies may include select samples of old adults that are more bilateral

\footnotetext{
Received March 23, 2012; revised July 21, 2012; accepted Sept. 9, 2012.

Author contributions: S.K., H.F., and L.B. designed research;S.K., H.F., and L.B. performed research; A.R. analyzed data; A.R. and L.B. wrote the paper.

This work was supported by grants from the Swedish Research Council and Swedish Brain Power, an Alexander von Humboldt Research Award, and a donation from the af Jochnich Foundation to L.B. We are grateful to Per Karlsson, Lars Farde, and Zsolt Cselenyi for their contributions to the PET data collection and analysis, and to Lars Nyberg for comments on an earlier version of the manuscript.

The authors declare no competing financial interests.

Correspondence should be addressed to Anna Rieckmann, Aging Research Center, Karolinska Institute, Gävlega$\tan 16$, S-113 30 Stockholm, Sweden. E-mail: rieckmann@nmr.mgh.harvard.edu.

DOI:10.1523/JNEUROSCI.1431-12.2012

Copyright $\odot 2012$ the authors $\quad 0270-6474 / 12 / 3217067-06 \$ 15.00 / 0$
}

already at outset, rather than increased bilaterality reflecting a change that occurs over the adult lifespan.

Striatal dopamine (DA) receptors play a crucial role in corticocortical loops that relay information to and from cortex via striatum and thalamus and are critically involved in several cognitive functions (Joel and Weiner, 2000). In an age-comparative study, we recently explored the association of reductions in striatal DA D1 receptor density to decreased frontoparietal functional connectivity and increased frontal bilateral connectivity in old age (Rieckmann et al., 2011). We found a pattern that suggested age-related reductions in frontoparietal connectivity are associated with reductions in striatal DA receptor densities, and that concomitant increases in cross-hemispheric prefrontal connectivity may compensate for these losses and contribute to maintaining performance during a WM task. Here, we sought to obtain direct experimental evidence for this interpretation by pharmacologically challenging the striatal DA D1 receptor system in young adults during fMRI and performance of a spatial WM task. We hypothesized that administration of a D1 receptor antagonist should induce reduced frontoparietal functional connectivity and increased bilateral prefrontal connectivity. Further, we explored whether the expected drug-related increase in prefrontal bilaterality is beneficial to performance under the influence of the antagonist. Such a pattern would support a compensatory interpretation of increased frontal bilaterality, and provide evidence for a causal link between striatal DA D1 receptors, functional connectivity, and WM.

\section{Materials and Methods}

Participants. Participants were 19 young adults (mean age: 25.16 years, age range: $22-30$ years, 9 female), from the same sample we have previously reported in age-comparative studies (Fischer et al., 2010; Bäckman et al., 2011; Rieckmann et al., 2011). All participants were right-handed, 
nonsmokers, and free from past or present drug or alcohol abuse, neuropsychiatric disorders, or brain damage.

Local ethics committees at Karolinska Hospital, Stockholm, Sweden, approved the study.

Procedure. Participants underwent two fMRI sessions, separated by, on average, $13 \mathrm{~d}(\mathrm{SD}=11)$. Before scanning, participants were injected with $0.5 \mathrm{mg}$ of SCH23390 ( $R$-(+)-7-chloro-8-hydroxy-3-methyl-1-phenyl2,3,4,5-tetrahydro- $1 \mathrm{H}$-3-benzazepine), a D1 receptor antagonist, in one session and with a placebo saline solution in the other session. The order of sessions was counterbalanced and participants were blind as to whether they received antagonist or placebo. In addition to fMRI acquisitions, positron emission tomography with ${ }^{11} \mathrm{C}$-labeled SCH23390 under antagonist and placebo was performed in 5 participants to assess D1 receptor occupancy of the antagonist. From these participants, it was estimated that the antagonist occupied on average $49.75 \%(S D=10.36)$ of caudate D1 receptors.

During each fMRI session, participants performed two runs of a spatial WM task. Stimuli were presented on a computer screen and viewed by participants via a mirror mounted on top of the head coil. Behavioral responses were collected with an MRI-compatible response pad. After completion of the last scan, participants were debriefed and received monetary compensation.

Cognitive task. Participants viewed a $4 \times 4$ grid in the center of a computer screen. For each trial, 4 (low load) or 6 (high load) circles consecutively appeared in different position on the grid at a rate of 900 $\mathrm{ms}$ with an interstimulus interval of $1100 \mathrm{~ms}$. A probe then appeared for $1750 \mathrm{~ms}$ to which participants had to indicate via button press whether or not a circle was presented in that position. The baseline was a perceptually similar $4 \times 4$ grid in which circles appeared in the center of the grid only and participants asked to press a button of their choice, with no demands on WM and modeled implicitly. The task was administered as a blocked design with three trials per block. Across two runs, participants completed 5 low-load and 5 high-load blocks, yielding a maximum accuracy score of 15 for each condition.

Accuracy was computed for each participant and condition and used to assess the correlation of performance change under antagonist (total accuracy $_{\text {antagonist }}-$ total accuracy placebo $_{\text {a }}$ ) to fMRI connectivity estimates. These correlations provide information about which changes in connectivity are related to WM performance under the influence of the DA antagonist.

MRI acquisition and analysis. Participants completed two fMRI sessions with identical acquisition parameters. Images were acquired on a 1.5 Tesla MRI system (Signa Excite HD Twinspeed, General Electric Medical Systems). For each assessment we collected two runs of 140 volumes, plus four dummy scans, of EPI data $(\mathrm{TR}=2.5 \mathrm{~s}$, TE $=40 \mathrm{~ms}$, flip angle $=90^{\circ}$, slice thickness $=4 \mathrm{~mm}$ with $0.5 \mathrm{~mm}$ gap, interleaved, FOV $=220 \mathrm{~mm}$ ). EPI images were coregistered with a T1-weighted structural image $($ SPGR sequence; $\mathrm{TR}=24 \mathrm{~ms}, \mathrm{TE}=6 \mathrm{~ms}$, flip angle $=$ $35^{\circ}$, slice thickness $=1.5 \mathrm{~mm}, \mathrm{FOV}=256 \mathrm{~mm}$ ).

All images were masked for non-brain tissue using FSL's Brain Extraction Tool (Smith, 2002). Motion correction using rigid-body transformations was performed on the functional images (MCFLIRT, Jenkinson et al., 2002). Further preprocessing steps included smoothing (full-width at half-maximum kernel $8 \mathrm{~mm}$ ), high-pass filtering and grand-mean scaling, based on standard procedures implemented in FSL's FEAT v5.98. The procedure for estimating first-level seed-based connectivity maps was identical to that of Rieckmann et al. (2011). In the current study, we focus on the frontoparietal network involved in cognitive control (Cabeza and Nyberg, 2000). The dorsolateral prefrontal cortex (DLPFC) is critically involved in this network. Given the spatial nature of the task, the right DLPFC was used as initiating seed (e.g., Smith et al., 1996). The seed was the same as used in Rieckmann et al. (2011) and based on peak activation (WM $>$ baseline) of an independent sample of 46 healthy adults performing an identical WM task (Brehmer et al., 2011). In MNI (Montreal Neurological Institute) space, peak coordinates for the initiating seed are $x=36, y=36, z=26$. The seed was coregistered to individual functional images and used to extract the time series for each preprocessed functional run.
The general linear model used to produce connectivity maps was based on three regressors: (1) the physiological regressor which was the time series of the initiating DLPFC seed, (2) the psychological regressor, which modeled high WM load as 1 and low WM load as -1 , and (3) the psychophysiological interaction (PPI), which is the interaction term of the first two regressors, centered at zero. The psychological regressor was convolved with a double-gamma hemodynamic response function. We also included 6 motion parameters as well as the time series for white matter, CSF, and global signal as confounders of no interest. The two runs of each fMRI assessment were averaged within persons to generate individual connectivity maps for each participant and session (antagonist and placebo). In subsequent analyses, we were mainly interested in group-level effects of the pharmacological challenge on functional connectivity of the right DLPFC, independent of load manipulation (i.e., regressor 1 , from here on referred to as load-independent findings). We previously found associations between D1 receptor density and functional connectivity estimates that were independent of WM load (Rieckmann et al., 2011). Additionally, we investigated antagonist effects on functional connectivity as a function of the load manipulation (i.e., regressor 3, from here on referred to as load-dependent findings) to allow for the possibility that the antagonist would affect the more demanding high-load WM condition more strongly.

For both load-independent and load-dependent higher-level analyses, we used a repeated-measures group level design with two connectivity maps (antagonist and placebo) for each participant and one regressor modeling the antagonist effect (antagonist $>$ placebo, antagonist $<$ placebo). Mean absolute displacement parameters at each scan were entered as an additional confounding variable to covary between-subject differences in movement, and their potential effect on seed-based connectivity estimates (Van Dijk et al., 2012). On average, participants did not differ in absolute mean displacement between scans (antagonist-placebo = $\left.0.001 \mathrm{~mm}(\mathrm{SD}=0.138), t_{(18)}<1\right)$. The functional data were coregistered to a standard brain template (MNI152) via the structural images.

The analysis yielded four whole-brain contrast maps of interest: loadindependent and load-dependent differences in functional connectivity of the right DLPFC for the two contrasts, antagonist $>$ placebo and antagonist $<$ placebo. The contrast maps were thresholded voxelwise at $p<0.01$ and corrected for multiple comparisons at the cluster level using Gaussian Random Field theory (GRF; $p<0.05$, leading to rejection of cluster sizes $<1124$ voxels). For visualization purposes, we also generated mean functional connectivity maps separately for placebo and antagonist conditions (Figs. 1, 2).

For subsequent correlational analyses with cognitive performance, peak voxels of each significant cluster were masked with a $6 \mathrm{~mm}$ region of interest (ROI). From these ROIs, parameter estimates were derived from each participant's contrast images (antagonist vs placebo). Standardized parameter estimates ( $Z$ scores) were then screened for outliers ( $\pm 2 \mathrm{SD})$. Outliers at this threshold were detected for 7 of 9 load-independent ROIs and for 14 of 18 load-dependent ROIs. We did not find more than one outlier per ROI. As the outliers were not the same individuals across ROIs, we excluded outliers ROI by ROI from the correlational analyses. Significance level for correlational analyses with performance were adjusted by number of ROIs tested to $p<0.05 / 9(=0.0056)$ for the load-independent analyses and to $p<0.05 / 18(=0.0028)$ for the load-dependent analyses.

\section{Results}

\section{Load-independent effects of antagonist on} functional connectivity

Figure 1 shows the mean functional connectivity maps under antagonist and placebo. Under antagonist, compared with placebo, we observed significantly increased functional connectivity of right DLPFC to a large frontal cluster, extending across hemispheres and with peaks in contralateral DLPFC (MNI $x, y, z$ coordinates: $-38,38,24)$, left precentral gyrus $(-58,8,22)$ and dorsomedial PFC (0, 50, 10; Fig. 1). Local functional connectivity (within right PFC; 32, 40, 26) was also increased with the antagonist. 

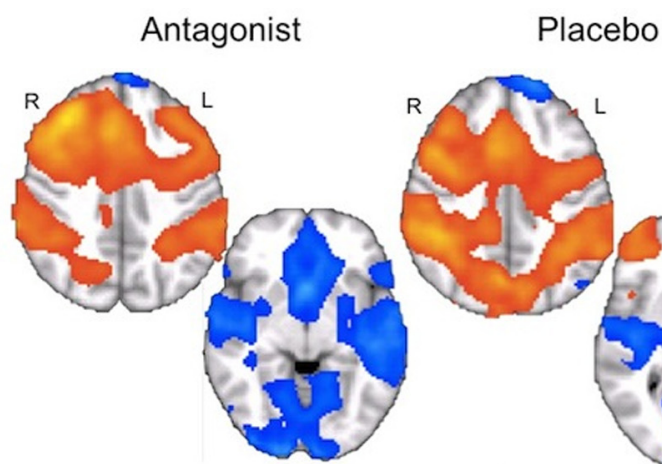

Placebo

Antagonist > Placebo
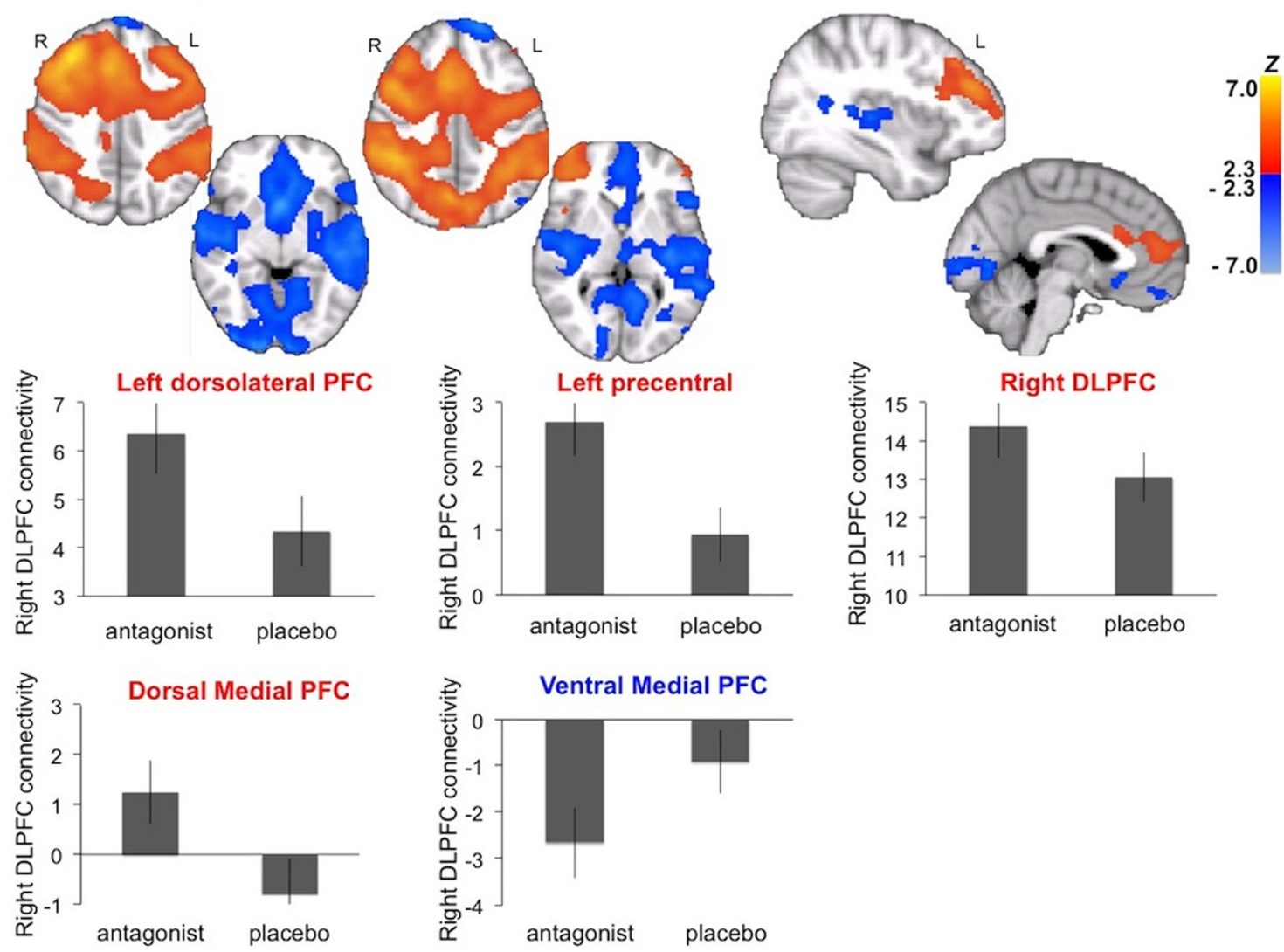

Figure 1. Load-independent connectivity maps, separately for each condition and for the contrast antagonist $>$ placebo. Bar graphs show average connectivity with right DLPFC (mean Z score with SE) for all frontal ROIs where a significant difference in connectivity between conditions was found.
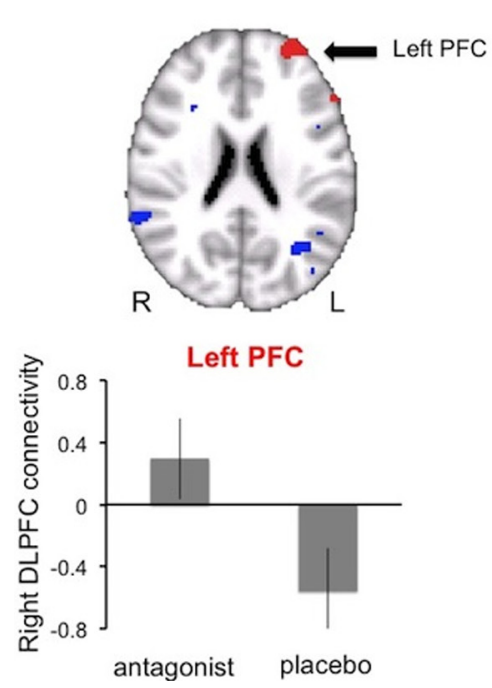

\section{Antagonist $>$ Placebo}
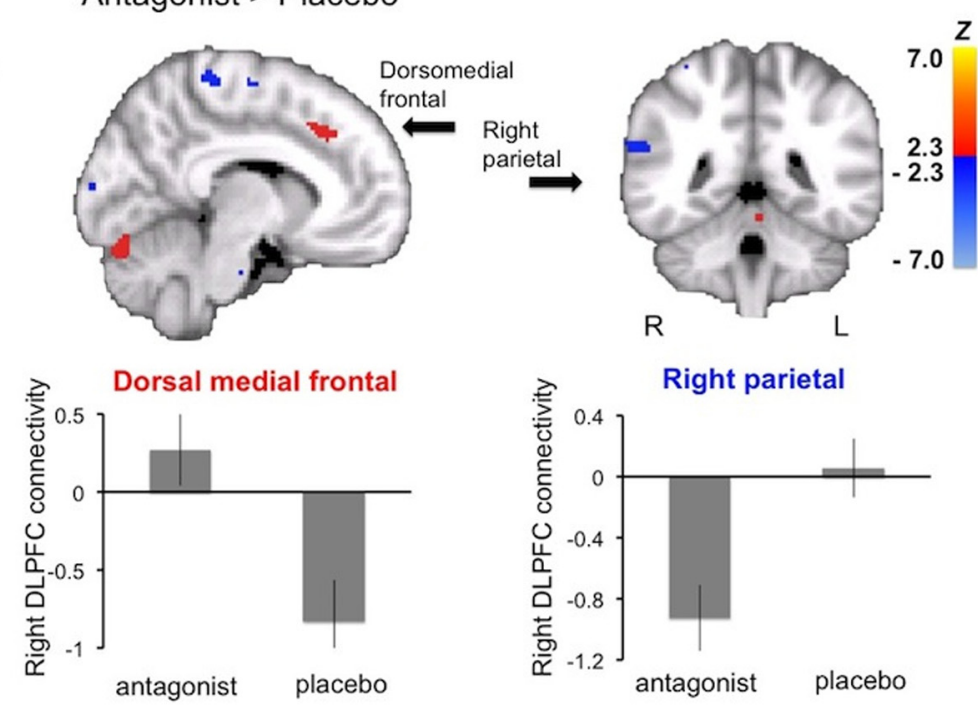

Figure 2. Contrast map for the comparison of load-dependent PPI connectivity maps between antagonist and placebo conditions. Bar graphs show average connectivity of right DLPFC (mean Z score and SE) for frontal and parietal ROIs. Negative effects in blue (antagonist < placebo) reflect reduced connectivity for the high-load compared with the low-load condition under the antagonist, whereas positive effects (antagonist $>$ placebo) reflect stronger connectivity during the high-load condition compared with the low-load condition under the antagonist.

For the reverse contrast, antagonist $<$ placebo, we found greater connectivity of right DLPFC to bilateral insular cortex/ middle temporal area, with peaks in left insula $(-38,-26,4)$ and right middle temporal gyrus $(42,-36,8)$. Greater connectivity under placebo was also observed to a ventral frontostriatal cluster with peaks in ventral striatum $(-12,18,-10)$, ventromedial PFC $(-10,46,-20$; Fig. 1), and occipital cortex $(-4,-96,-4)$. As shown in Figure 1 for the ventromedial PFC, all clusters in this contrast reflected greater negative connectivity during the antagonist. 
Load-dependent (PPI) effects of antagonist on functional connectivity For the load-dependent PPI analysis, a negative parameter estimate (antagonist $<$ placebo) reflects reduced connectivity for the high-load compared with the low-load condition under the antagonist, whereas a positive effect (antagonist $>$ placebo) reflects stronger connectivity during the high-load condition with the antagonist.

There were no significant differences between placebo and antagonist in terms of load-dependent connectivity changes (PPI) at the GRF-corrected significance level. We did find effects of the antagonist at a voxelwise threshold of $p<0.01$ without cluster size thresholding, but these should be interpreted cautiously (cluster sizes between 60 and 404 voxels; Fig. 2). Notably, in this more liberal analysis we observed drug-related connectivity decreases of right DLPFC to right parietal cortex (supramarginal gyrus; 64, -44 , $22)$, and brainstem $(2,-20,-32)$. Although not completely overlapping, we previously observed positive associations between dopamine receptor density and connectivity of the right DLPFC to nearby voxels (Rieckmann et al., 2011, supramarginal gyrus: $60,-30,34$; brainstem: 8 , $-28,-10)$. A similar pattern was observed for motor areas, including peaks in right $(48,0,54)$ and left $(-22,-12,62)$ precentral gyri and supplementary motor cortex $(4,0,60)$, postcentral gyrus $(-8$, $-34,66)$ as well as occipital areas $(20,-88,12 ;-48,-80,4)$ and parahippocampal gyrus $(22,-10,-28)$.

For the reverse contrast, there was increased connectivity under the antagonist of right DLPFC to dorsomedial PFC (10, 40, $36)$, left PFC $(-28,56,22)$, thalamus $(-26,-34,10)$, anterior middle temporal cortex $(-62,-6,-12)$, and five small clusters in cerebellum. Although we found both decreased frontoparietal and increased frontal bilaterality under the antagonist in the load-dependent analyses, we did not obtain a significant correlation between antagonist effects on frontal bilaterality and frontoparietal connectivity here $(p>0.10)$.

Previous research has shown that pharmacological manipulations of the DA system may affect frontostriatal connectivity (Kelly et al., 2008; Nagano-Saito et al., 2008; Wallace et al., 2011). In line with these studies, we observed functional connectivity of right DLPFC to caudate under placebo, but not under the influence of the antagonist. However, in the current study the difference between conditions was not statistically reliable $(p=0.127$, uncorrected).

\section{Correlations between cognitive performance and functional connectivity}

For all nine clusters identified in the load-independent analysis, we computed correlations between performance change (accuracy antagonist - accuracy placebo) and connectivity estimates ( $Z$ score) around the cluster peak. We found that a greater increase in connectivity between right and left DLPFC was related to maintaining performance under the antagonist compared with placebo $(r=0.66, p=0.002)$. When performance was estimated separately for the low-load and highload conditions, we found a significant correlation between increased bilateral frontal connectivity and performance in the high-load condition ( $r=0.72, p=0.001$; Fig. 3$)$, but not in the low-load condition $(r=0.32, p=0.17)$. Similar associations were observed for ventromedial PFC, where we found decreased connectivity with the antagonist compared with placebo at the group level. Here, functional connectivity was negatively related to the performance change for overall accuracy at trend level $(r=-0.57, p=0.01)$ and significantly so for the high-load condition $(r=-0.68, p=0.001$; Fig. 3$)$. Together, individuals who performed better on the WM task under the antagonist engaged more bilateral dorsal PFC, while decreasing connectivity with the ventromedial PFC, particularly under the more demanding WM condition.

Previous research has shown that effects of dopamine agonists are dependent on performance under placebo conditions (Cools and D'Esposito, 2011). In the current study, placebo performance on the WM task predicted behavioral perfor-

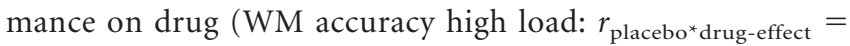
$-0.62, p<0.006$; RT low load: $r_{\text {placebo }{ }^{\star} \text { drug-effect }}=-0.71, p<$ 0.006; RT high load: $\left.r_{\text {placebo }}{ }_{\text {drug-effect }}=-0.71, p<0.006\right)$ as well as change in frontal connectivity to MPFC (WM high load: $\left.r_{\text {accuracy }}{ }_{\text {DLPFC-medialPFC }}=0.75, p<0.006\right)$, such that individuals with low performance under placebo showed an improvement in behavior as well as stronger anticorrelations to MPFC under the antagonist. For the ROIs derived from the 
load-dependent analyses, there were no significant correlations between performance and functional connectivity.

\section{Discussion}

We recently demonstrated an association between age-related reductions in striatal D1 receptor density and reduced frontoparietal connectivity during a WM task (Rieckmann et al., 2011). We also found increased frontal bilaterality in old compared with young adults, which may reflect a shift in response to impaired frontoparietal pathways. In the current study, we sought to provide evidence for a causal relationship between D1 receptor depletion and functional connectivity of the DLPFC during WM by administering an antagonist to young adults. The most striking observation was that participants showed increased frontal connectivity of right DLPFC to left DLPFC, dorsomedial PFC, and precentral gyrus under the influence of the antagonist. Critically, increased frontal connectivity of right to left DLPFC was associated with less performance change from the antagonist. In other words, those individuals who showed greater frontal bilaterality under the antagonist were also most likely to maintain performance during the pharmacological challenge. An additional load-dependent PPI analysis found that increased frontal bilaterality with the antagonist was most pronounced for the more difficult high-load condition.

Interestingly, increased frontal bilaterality was restricted to a dorsal bilateral frontal network, whereas ventromedial frontal areas were negatively correlated with right DLPFC under the antagonist. The negative connectivity with ventromedial PFC likely reflects anticorrelations of the frontoparietal network to parts of the default mode network (Fox et al., 2005). Anticorrelations during the antagonist increased in spatial extent compared with placebo from posterior cingulate to occipital areas, from angular gyrus to middle temporal gyrus/posterior insular, and from medial PFC to ventromedial areas and ventral striatum (Fig. 1). Stronger anticorrelations between DLPFC and ventromedial PFC under the antagonist contributed to maintaining performance relative to placebo (Fig. 3), and a correlation with placebo performance level suggested that greater anticorrelations and better performance on drug were related to lower placebo performance. In line with the idea of an inverted $\mathrm{U}$-shape relation between dopamine level under placebo and performance on drug, this pattern would indicate that subjects were on the higher end of the dopamine curve under placebo, so that an antagonist may in fact be beneficial to performance and network connectivity. However, in our previous study we did not observe a correlation between DA level as measured with PET and anticorrelations of DLPFC to ventromedial PFC. The pattern of decreased anticorrelations in young adults under antagonist does also not mirror previous observations in old adults, which have shown agerelated reductions (Sambataro et al., 2010). This suggests that altered functional connectivity in older adults is a multifaceted phenomenon, with different age-related brain changes mediating functioning of different brain networks.

We did not find strong evidence of decreased frontoparietal connectivity under the antagonist. The load-dependent analysis yielded a decrease in the frontoparietal load effect with the antagonist compared with placebo, suggesting a reduction in frontoparietal connectivity during the high-load condition (Fig. 2). However, this effect was relatively weak and not related to any performance measure.

\section{Increased frontal bilateral connectivity under DA antagonist:} sign of compensation?

An intriguing finding from this study is the increased bilateral coupling within frontal cortex in young adults under the pharmacological challenge. This temporary increase in frontal connectivity is interesting to view in relation to studies suggesting that age-related increases in frontal bilateral activation reflect sampling bias (Nyberg et al., 2010), or that increases in bilateral frontal connectivity are associated with age-related structural changes (Davis et al., 2012). We provide novel evidence that the prefrontal shift is not unique to older adults, or necessarily associated with age-related structural decline, but can be elicited in young adults by pharmacologically challenging corticostriatal pathways with a D1 receptor antagonist. We also show that increased bilateral recruitment of a dorsal frontal network was related to reduced accuracy decrements under the antagonist and may therefore serve compensatory purposes.

The term compensation often carries the connotation of rewiring or reorganization of the brain that accompanies successful aging and combats structural losses (e.g., Reuter-Lorenz, 2002). The current data suggest that rather than reorganization, compensation can reflect a shift in brain pathways in response to neurocognitive challenges. On this view, different brain circuits, for example a frontoparietal network and a bilateral dorsofrontal network, are available for task performance. Although the frontoparietal network appears to be the most efficient selection in healthy young adults, an alternate bilateral frontal pathway may be recruited when the frontoparietal pathway is functionally impaired. Thus, successful cognitive performance may depend not on reorganization of the brain, but on the ability to flexibly select between alternative neural pathways, regardless of age (Lindenberger et al., 2012). However, it remains to be determined which genetic and environmental factors promote compensatory recruitment of cross-hemispheric frontal pathways in response to neurocognitive challenges of the frontoparietal circuitry. As is typical also for age-comparative studies (e.g., Grady et al., 2005; Gutchess et al., 2005), we found considerable individual differences in the extent of increased drug-related frontal bilaterality and its protective effect against performance changes (Fig. 3). Our data suggest that individual differences in neurotransmitter functioning of specific brain pathways are critically related to differences in functional connectivity during task performance. As subtle biochemical changes likely precede or co-occur with age-related gray-matter atrophy and white-matter changes, future research should aim to differentiate contributions of agerelated changes in neurotransmitter functioning and brain morphology to functional connectivity of specific pathways and networks.

\section{Caveats}

Although the strong positive correlations between increased frontal bilaterality and WM performance under the antagonist provide support for compensation, some aspects of our results remain inconsistent with such an interpretation. Based on our earlier findings of an association between frontoparietal connectivity and striatal D1 receptor density, we expected a drug-related decrease in connectivity in this pathway (Rieckmann et al., 2011). Moreover, in case of compensatory recruitment of a frontal bilateral network under the antagonist, we would have expected a concomitant increase in frontal bilaterality in response to frontoparietal decreases. However, drug-related frontoparietal connectivity reductions were restricted to a small cluster and only found in the load-dependent analysis, and also located more pos- 
terior $(x=64, y=-44, z=22)$, to our previously reported cluster of frontoparietal connectivity $(x=60, y=-30, z=34)$, whereas frontal bilaterality was widespread and generalized to both load-independent and load-dependent analyses. In the absence of a strong frontoparietal connectivity reduction under the antagonist, it is difficult to specify what the increased frontal bilaterality compensates for. That said, we previously demonstrated a negative correlation between frontoparietal connectivity and frontal bilaterality in a larger sample with a greater range in connectivity (Rieckmann et al., 2011). The absence of an association between frontoparietal connectivity and frontal bilaterality observed here may reflect difficulties in detecting complex correlations between two difference scores.

\section{Conclusions}

We show that administration of a D1 receptor antagonist in healthy young adults results in increased functional connectivity between right DLPFC and dorsomedial and left frontal areas. This pattern resembles findings from age-comparative studies that have shown increased frontal bilateral activation and connectivity in old compared with young adults. Increased frontal bilaterality in old adults remains a widely discussed topic in the cognitive neuroscience of aging. Here, we provide novel evidence that frontal bilateral connectivity can be demonstrated in healthy young adults with a D1 receptor antagonist. We also show that increased bilateral connectivity is beneficial to maintaining WM performance under a DA antagonist, suggesting a compensatory response to a neurocognitive challenge. Our data add to previous suggestions that increased frontal bilaterality could reflect sampling bias or structural damage, as we show that this pattern can be temporarily induced in healthy young adults. The results suggest that subtle changes in the DA system can temporarily alter the recruitment of specific brain networks.

\section{References}

Bäckman L, Karlsson S, Fischer H, Karlsson P, Brehmer Y, Rieckmann A, MacDonald SW, Farde L, Nyberg L (2011) Dopamine D(1) receptors and age differences in brain activation during working memory. Neurobiol Aging 32:1849-1856. CrossRef Medline

Brehmer Y, Rieckmann A, Bellander M, Westerberg H, Fischer H, Bäckman L (2011) Neural correlates of training-related working-memory gains in old age. Neuroimage 58:1110-1120. CrossRef Medline

Cabeza R, Nyberg L (2000) Imaging cognition II: an empirical review of 275 PET and fMRI studies. J Cogn Neurosci 12:1-47. CrossRef Medline

Cools R, D'Esposito M (2011) Inverted-U-shaped dopamine actions on human working memory and cognitive control. Biol Psychiatry 69:e113e125. CrossRef Medline

Davis SW, Kragel JE, Madden DJ, Cabeza R (2012) The architecture of cross-hemispheric communication in the aging brain: linking behavior to functional and structural connectivity. Cereb Cortex 22:232-242. CrossRef Medline

Fischer H, Nyberg L, Karlsson S, Karlsson P, Brehmer Y, Rieckmann A, MacDonald SW, Farde L, Bäckman L (2010) Simulating neurocognitive aging: effects of a dopaminergic antagonist on brain activity during working memory. Biol Psychiatry 67:575-580. CrossRef Medline

Fox MD, Snyder AZ, Vincent JL, Corbetta M, Van Essen DC, Raichle ME (2005) The human brain is intrinsically organized into dynamic, anticorrelated functional networks. Proc Natl Acad Sci U S A 102:9673-9678. CrossRef Medline

Goh JO (2011) Functional dedifferentiation and altered connectivity in older adults: neural accounts of cognitive aging. Aging Dis 2:30-48. Medline

Grady CL, McIntosh AR, Craik FI (2005) Task-related activity in prefrontal cortex and its relation to recognition memory performance in young and old adults. Neuropsychologia 43:1466-1481. CrossRef Medline

Gutchess AH, Welsh RC, Hedden T, Bangert A, Minear M, Liu LL, Park DC (2005) Aging and the neural correlates of successful picture encoding: frontal activations compensate for decreased medial-temporal activity. J Cogn Neurosci 17:84-96. CrossRef Medline

Jenkinson M, Bannister P, Brady M, Smith S (2002) Improved optimization for the robust and accurate linear registration and motion correction of brain images. Neuroimage 17:825-841. CrossRef Medline

Joel D, Weiner I (2000) The connections of the dopaminergic system with the striatum in rats and primates: an analysis with respect to the functional and compartmental organization of the striatum. Neuroscience 96:451-474. CrossRef Medline

Kelly AMC, Uddin LQ, Biswal BB, Castellanos FX, Milham MP (2008) Competition between functional brain networks mediates behavioral variability. Neuroimage 39:527-537. CrossRef Medline

Lindenberger U, Burzynska AZ, Nagel IE (2012) Heterogeneity in frontallobe aging. In: Principles of frontal lobe functions, Ed 2 (Stuss DT, Knight RT, eds). New York: Oxford UP.

Morcom AM, Friston KJ (2012) Decoding episodic memory in ageing: a Bayesian analysis of activity patterns predicting memory. Neuroimage 59:1772-1782. CrossRef Medline

Nagano-Saito A, Leyton M, Monchi O, Goldberg YK, He Y, Dagher A (2008) Dopamine depletion impairs frontostriatal functional connectivity during a set-shifting task. J Neurosci 28:3679-3706.

Nagel IE, Preuschhof C, Li SC, Nyberg L, Bäckman L, Lindenberger U, Heekeren HR (2011) Load modulation of BOLD response and connectivity predicts working memory performance in younger and older adults. J Cogn Neurosci 23:2030-2045. CrossRef Medline

Nyberg L, Salami A, Andersson M, Eriksson J, Kalpouzos G, Kauppi K, Lind J, Pudas S, Persson J, Nilsson LG (2010) Longitudinal evidence for diminished frontal cortex function in aging. Proc Natl Acad Sci U S A 107: 22682-22686. CrossRef Medline

Rajah MN, D'Esposito M (2005) Region-specific changes in prefrontal function with age: a review of PET and fMRI studies on working and episodic memory. Brain 128:1964-1983. CrossRef Medline

Reuter-Lorenz P (2002) New visions of the aging mind and brain. Trends Cogn Sci 6:394-400. CrossRef Medline

Rieckmann A, Karlsson S, Fischer H, Bäckman L (2011) Caudate dopamine $\mathrm{D} 1$ receptor density is associated with individual differences in frontoparietal connectivity during working memory. J Neurosci 31:14284-14290. CrossRef Medline

Sambataro F, Murty VP, Callicott JH, Tan HY, Das S, Weinberger DR, Mattay VS (2010) Age-related alterations in default mode network: impact on working memory performance. Neurobiol Aging 31:839-852. CrossRef Medline

Smith EE, Jonides J, Koeppe RA (1996) Dissociating verbal and spatial working memory using PET. Cereb Cortex 6:11-20. CrossRef Medline

Smith SM (2002) Fast robust automated brain extraction. Hum Brain Mapp 17:143-155. CrossRef Medline

Spreng RN, Wojtowicz M, Grady CL (2010) Reliable differences in brain activity between young and old adults: a quantitative meta-analysis across multiple cognitive domains. Neurosci Biobehav Rev 34:1178-1194. CrossRef Medline

Van Dijk KR, Sabuncu MR, Buckner RL (2012) The influence of head motion on intrinsic functional connectivity MRI. Neuroimage 59:431-438. CrossRef Medline

Wallace DL, Vytlacil JJ, Nomura EM, Gibbs SEB, D’Esposito M (2011) The dopamine agonist bromocriptine differentially affects frontostriatal functional connectivity during working memory. Front Hum Neurosci 5:1-6. CrossRef 